\title{
Effect of Fluctuating Aerosol Concentrations on the Aerosol Distributions in a Turbulent Jet
}

\author{
Hailin Yang ${ }^{1}$, Jianzhong Lin $^{1 *}$, Tatleung Chan ${ }^{2}$ \\ ${ }^{1}$ Department of Mechanics, State Key Laboratory of Fluid Power and Mechatronic Systems, Zhejiang University, \\ Hangzhou 310027, China \\ ${ }^{2}$ Department of Mechanical Engineering, The Hong Kong Polytechnic University, Kowloon, Hong Kong
}

\begin{abstract}
We apply the Reynolds averaging method to derive the averaged particle general dynamic equation (APGDE), which models the effect of fluctuations in aerosol concentrations on coagulation, referred to as the fluctuating coagulation term (FCT), based on the turbulent kinetic energy. The APGDE is numerically solved using the Taylor-series expansion method of moments for a turbulent jet; the equation is then validated by comparing some of the results with experimental values. Obtaining the distribution and evolution of the aerosol particle number concentration, mass concentration, mean diameter and geometric standard deviation both when the FCT is incorporated and when it is excluded, we find that the contribution of fluctuating concentrations to the coagulation becomes a factor when $x / D=5$ and reaches a stable state when $x / D=20$. When the FCT is considered, the number concentration, mean diameter and geometric standard deviation are clearly lower, larger and greater, respectively. The fluctuating concentrations intensify the coagulation, reduce the number concentration and increase the mean diameter. Therefore, such fluctuations in a turbulent jet cannot be neglected.
\end{abstract}

Keywords: Aerosol particles; Fluctuating aerosol concentration; Fluctuating coagulation; Aerosol distribution; Turbulent jet.

\section{INTRODUCTION}

Air quality is related to the amount of aerosols in the atmosphere. To understand the number and distribution of aerosols requires understanding the generation and motion and dynamics of aerosols. So the solution to the aerosol particle general dynamic equation (PGDE) is required. PGDE proposed by Smoluchowski is a fundamental equation describing the evolution of aerosol particle distribution, and made up of unsteady, convective, diffusion and source terms. The source terms are contributed by many physical and chemical processes including aerosol particle coagulation, growth, nucleation, condensation and so on (Lesniewski and Friedlander, 1998; Zhou and Chan, 2011; Chan et al., 2018). In the aerosol flow, the turbulent motion is more common (Zhu et al., 2018). The effect of turbulence on the source terms has been investigated extensively.

Reade and Collins (2000) simulated the fluid flow and particle motion using direct numerical simulation method and statistical method, respectively, and showed that, in general, the degree of particle preferential concentration increases

\footnotetext{
* Corresponding author.

Tel.: +86 57187952882

E-mail address: mecjzlin@public.zju.edu.cn
}

with increasing Reynolds number. Zajchik and Solov'Ev (2002) investigated the particle collision and coagulation due to Brownian and turbulent effect, and found that the coagulation kernel varies with increasing particle sizes while passing through a minimum in the region transitioning from the Brownian continual to turbulent mode. Chun et al. (2005) solved the equation of aerosol size distribution function to study the clustering of aerosol particles in isotropic turbulence, and showed that clustering of aerosol particles increases the rate of coagulation or coalescence. Das and Garrick (2010) investigated the growth and Brownian coagulation of aerosol particle in the turbulent jet flow calculated by large eddy simulation, and showed that the unresolved small-scale fluctuations can both enhance and restrain particle growth, however, the predominant influence is to reduce particle growth. Gan et al. (2010) studied the turbulent coagulation and breakage of particle in the turbulent jet flow, and found that the polydispersity and particle diameter are very large in the shear layers at the upstream and in the core of vortex structures at the downstream. Loeffler et al. (2011) used the subgrid-scale modeling of mean concentration coagulation term and obtained the formation and growth of aerosol particle in turbulent jet, they suggested that neglect of the unresolved particle-particle interactions may enhance the particle growth rate with increasing the precursor concentration. Yin and Liu (2014) showed that the interaction of the two jets and turbulence eddy structures rolling up, paring and shedding in 
flow sharply affects particles' number concentration. Particle diameter grows quickly at the interfaces of jets. Liu and Chan (2017) combined the Monte Carlo method and PGDE to simulate the complex aerosol dynamics in turbulent flows with considering the turbulent effect on the convective and diffusion terms of the aerosol particle, and it is shown that Reynolds number has significant impact on the aerosol particle coagulation. Rittler et al. (2017) simulated the aerosol synthesis from flame spray pyrolysis with considering the turbulent effect on the convective and nucleation terms of the particle, and showed that turbulent mixing leads to a broader size distribution of the primary and aggregate particle diameters.

All the references mentioned above refer to the effect of fluid fluctuation on the aerosol particles in the turbulent flow. However, the concentration fluctuation of aerosol particles also has the effect on the aerosol particle distributions. From a theoretical point of view, the physical quantities of fluid and particle could be written as the sum of mean and fluctuating components. Therefore, the averaged particle general dynamic equation (APGDE) can be derived using Reynolds averaging method, and two new terms appear in the APGDE (Friedlander, 2000). One of those is the contribution to coagulation resulting from the fluctuating concentrations of aerosol particles, referred as the fluctuating coagulation term (FCT). The FCT is very complex and used to be neglected because of lacking available theory and model. Lin et al. (2016) modeled the FCT and applied it to a turbulent pipe flow. However, the pipe flow is a kind of confined flow with effect of wall. In the present study, therefore, in order to explore the effect of fluctuating concentrations on the aerosol particle coagulation as well as the distribution in the free shear flow, the FCT is analyzed and modeled based on the turbulent kinetic energy in a turbulent jet. Then the APGDE is solved numerically combined with the Reynolds-averaged Navier-Stokes equation and $k-\varepsilon$ equation. The aerosol particle mass concentration, number concentration, mean diameter and geometric standard deviation are simulated numerically.

\section{MATHEMATICAL FORMULATION AND MODELS}

\section{Particle General Dynamic Equation for Turbulent Flows}

In the present study, we explore the combined effects of convection, diffusion and coagulation on the evolution of aerosol particles. The PGDE which governs the evolution of aerosol particles in the flow is given:

$$
\begin{aligned}
& \frac{\partial n(v, t)}{\partial t}+\boldsymbol{u} \nabla \cdot n(v, t)-\nabla \cdot D \nabla n(v, t)= \\
& \frac{1}{2} \int_{0}^{v} \beta\left(v_{1}, v-v_{1}\right) n\left(v_{1}, t\right) n\left(v-v_{1}, t\right) d v_{1} \\
& -\int_{0}^{\infty} \beta\left(v_{1}, v\right) n\left(v_{1}, t\right) n(v, t) d v_{1}
\end{aligned}
$$

where $n(v, t)$ is the particle size distribution function; $\boldsymbol{u}$ is the fluid velocity vector; $\beta\left(v_{1}, v\right)$ is the coagulation kernel for two particles of volume $v$ and $v_{1} ; D$ is the particle diffusion coefficient and can be given by:

$$
\begin{aligned}
& D=\frac{k_{B} T C_{c}}{3 \pi \mu d_{p}} ; C_{c}=1+K n\left[1.257+0.40 \exp \left(-\frac{1.10}{K n}\right)\right] \\
& K n=2 \lambda / d_{p}
\end{aligned}
$$

where $T$ is the temperature; $k_{B}$ is the Boltzmann constant; $\mu$ is the dynamic viscosity of fluid; $d_{p}$ is the particle diameter and $K n$ is the Knudsen number (the ratio of gas molecular mean free path to the particle radius).

To obtain the PGDE in turbulent flow, the fluid velocity and particle size distribution function are decomposed into the mean and fluctuating components:

$\boldsymbol{u}=\overline{\boldsymbol{u}}+\boldsymbol{u}^{\prime}, n=\bar{n}+n^{\prime}$

Substituting Eq. (3) into Eq. (1) and averaging with respect to time, we obtain the averaged particle general dynamic equation:

$$
\begin{aligned}
& \frac{\partial \bar{n}(v, t)}{\partial t}+\overline{\boldsymbol{u}} \nabla \cdot \bar{n}(v, t)-\nabla \cdot D \nabla \bar{n}(v, t)+\nabla \cdot \overline{n^{\prime} \boldsymbol{u}^{\prime}} \\
& =\frac{1}{2} \int_{0}^{v} \beta\left(v_{1}, v-v_{1}\right) \bar{n}\left(v_{1}, t\right) \bar{n}\left(v-v_{1}, t\right) d v_{1} \\
& -\int_{0}^{\infty} \beta\left(v_{1}, v\right) \bar{n}\left(v_{1}, t\right) \bar{n}(v, t) d v_{1} \\
& +\frac{1}{2} \int_{0}^{v} \beta\left(v_{1}, v-v_{1}\right) \overline{n^{\prime}\left(v_{1}, t\right) n^{\prime}\left(v-v_{1}, t\right)} d v_{1} \\
& -\int_{0}^{\infty} \beta\left(v_{1}, v\right) \overline{n^{\prime}\left(v_{1}, t\right) n^{\prime}(v, t)} d v_{1}
\end{aligned}
$$

where the last term on the left-hand side can be changed into the form of turbulent diffusion of the quantity $\bar{n}$ :

$\overline{n^{\prime} \boldsymbol{u}^{\prime}}=-v_{t} \frac{\partial \bar{n}}{\partial x_{i}}$

where $v_{t}$ is the turbulent viscosity and can be obtained by solving the equations of turbulent flow.

The third and fourth terms on the right-hand side of Eq. (4) denote the contribution to coagulation resulting from the fluctuating concentrations (the fluctuating coagulation term), which used to be neglected before. Here we make a following assumption based on the relationship between correlation of fluctuating concentration and the mean concentration, mean flow kinetic energy and turbulent kinetic energy as:

$\overline{n^{\prime}\left(v_{i}, t\right) n^{\prime}\left(v_{i}, t\right)}=\chi_{t} \bar{n}\left(v_{i}, t\right) \bar{n}\left(v_{j}, t\right)$

where the factor $\chi_{t}$ is referred to as number concentration fluctuation intensity:

$\chi_{t}=k /\left(\bar{u}_{i}^{2} / 2+k\right)$ 
in which $k$ is the turbulent kinetic energy; $\overrightarrow{u_{i}{ }^{2}}$ is the mean flow kinetic energy.

Substituting Eq. (5) and Eq. (6) into Eq. (4), we obtain the APGDE:

$$
\begin{aligned}
& \frac{\partial \bar{n}(v, t)}{\partial t}+\bar{u} \nabla \cdot \bar{n}(v, t)-\nabla \cdot\left(D+v_{t}\right) \nabla \bar{n}(v, t) \\
& =\frac{1}{2} \int_{0}^{v} \beta\left(v_{1}, v-v_{1}\right)\left(1+\chi_{t}\right) \bar{n}\left(v_{1}, t\right) \bar{n}\left(v-v_{1}, t\right) d v_{1} \\
& -\int_{0}^{\infty} \beta\left(v_{1}, v\right)\left(1+\chi_{t}\right) \bar{n}\left(v_{1}, t\right) \bar{n}(v, t) d v_{1}
\end{aligned}
$$

\section{Moment Equation and Moment Method}

Numerical method has to be employed because of the complexity of the PGDE. These methods include the moment method (Yu et al., 2006; Lin et al., 2016; Lai et al., 2018), the sectional method (Chen et al., 2015), the Monte Carlo method (Liu and Chan, 2018) and so on. The moment method is based on the following moment equation:

$m_{j}=\int_{0}^{\infty} v^{j} \bar{n}(v) d v$

where $j$ represents the order of the moment. Here we focus on the first three orders $(j=0,1,2)$. The zeroth moment $m_{0}$ and first moment $m_{1}$ are proportional to the particle number concentration and total particle mass, respectively. The second moment $m_{2}$ corresponds to the mean particle size and polydispersity.

Based on Eq. (9), Eq. (8) can be transformed into an ordinary differential equation with respect to the moment $m_{j}$ :

$$
\begin{aligned}
& \frac{\partial m_{j}}{\partial t}+\overline{\boldsymbol{u}} \nabla m_{j}-\nabla \cdot\left(D+v_{t}\right) \nabla m_{j} \\
& =\frac{1}{2} \int_{0}^{\infty} \int_{0}^{\infty}\left[\left(v+v_{1}\right)^{j}-v^{j}-v_{1}^{j}\right]\left(1+\chi_{t}\right) \beta\left(v_{1}, \mathrm{v}\right) \bar{n}\left(v_{1}, t\right) \bar{n}(v, t) d v_{1} d v
\end{aligned}
$$

Thus, we have the transport equations of first three moments:

$$
\begin{aligned}
& \frac{\partial m_{0}}{\partial t}+\overline{\boldsymbol{u}} \nabla m_{0}-\nabla \cdot\left(D+v_{t}\right) \nabla m_{0} \\
& =\frac{1}{2} \int_{0}^{\infty} \int_{0}^{\infty}\left(1+\chi_{t}\right) \beta\left(v_{1}, \mathrm{v}\right) \bar{n}\left(v_{1}, t\right) \bar{n}(v, t) d v_{1} d v \\
& \frac{\partial m_{1}}{\partial t}+\overline{\boldsymbol{u}} \nabla m_{1}-\nabla \cdot\left(D+v_{t}\right) \nabla m_{1}=0 \\
& \frac{\partial m_{2}}{\partial t}+\overline{\boldsymbol{u}} \nabla m_{2}-\nabla \cdot\left(D+v_{t}\right) \nabla m_{2} \\
& =\frac{1}{2} \int_{0}^{\infty} \int_{0}^{\infty} v v_{1}\left(1+\chi_{t}\right) \beta\left(v_{1}, \mathrm{v}\right) \bar{n}\left(v_{1}, t\right) \bar{n}(v, t) d v_{1} d v
\end{aligned}
$$

The particle coagulation kernel $\beta\left(v_{1}, v\right)$ should be given in advance before Eq. (11) is solved. Here the Brownian coagulation in free molecule regime is considered for the nanoparticles (Friedlander, 2000):

$$
\beta_{B, F}=A_{1}\left(\frac{1}{v}+\frac{1}{v_{1}}\right)\left(v^{1 / 3}+v_{1}^{1 / 3}\right)^{2} ; A_{1}\left(\frac{3}{4 \pi}\right)^{1 / 6}\left(\frac{6 k_{B} T}{\rho_{p}}\right)^{1 / 2}
$$

where $\rho_{p}$ is the particle density.

Substituting Eq. (12) into Eq. (11), we can obtain the transport equations which include fractional order and negative order moments and are not closure. To dispose the surplus moments and get closured equations, we use the Taylor-series expansion method of moment (TEMOM) (Yu et al., 2008) to expand $v^{j}$ about $v=w$ (where $w=m_{1} / m_{0}$ represent the volume of particle) and remain the first three term of Taylor series. Thus, arbitrary index $v^{j}$ can be transformed into $v^{0}, v^{1}, v^{2}$ using following expression:

$$
\begin{aligned}
v^{j} & =\left(\frac{w^{j-2} j^{2}}{2}-\frac{w^{j-2} j}{2}\right) v^{2}+\left(-w^{j-1} j^{2}+2 w^{j-1} j\right) v \\
& +\left(w^{j}+\frac{w^{j} j^{2}}{2}-\frac{3 w^{j} j}{2}\right)
\end{aligned}
$$

Above expression can be transformed into moment style using Eq. (9):

$$
\begin{aligned}
m_{j} & =\left(\frac{w^{j-2} j^{2}}{2}-\frac{w^{j-2} j}{2}\right) m_{2}+\left(-w^{j-1} j^{2}+2 w^{j-1} j\right) m_{1} \\
& +\left(w^{j}+\frac{w^{j} j^{2}}{2}-\frac{3 w^{j} j}{2}\right) m_{0}
\end{aligned}
$$

Substituting Eqs. (9), (12) and (13) into Eq. (11), we get:

$\frac{\partial m_{0}}{\partial t}+\overline{\boldsymbol{u}} \nabla m_{0}-\nabla \cdot\left(D+v_{t}\right) \nabla m_{0}$

$=\sqrt{2} A_{1}\left(1+\chi_{t}\right) \frac{65 m_{2}{ }^{2} m_{0}{ }^{23 / 6}-1210 m_{2} m_{1}{ }^{2} m_{0}{ }^{17 / 6}-9223 m_{1}{ }^{4} m_{0}{ }^{11 / 6}}{5184 m_{1}{ }^{23 / 6}}$

$\frac{\partial m_{1}}{\partial t}+\overline{\boldsymbol{u}} \nabla m_{1}-\nabla \cdot\left(D+v_{t}\right) \nabla m_{1}=0$

$$
\begin{aligned}
& \frac{\partial m_{0}}{\partial t}+\overline{\boldsymbol{u}} \nabla m_{0}-\nabla \cdot\left(D+v_{t}\right) \nabla m_{0} \\
& =\sqrt{2} A_{1}\left(1+\chi_{t}\right) \frac{-701 m_{2}{ }^{2} m_{0}{ }^{11 / 6}-4210 m_{2} m_{1}{ }^{2} m_{0}{ }^{5 / 6}-6859 m_{1}{ }^{4} m_{0}{ }^{-1 / 6}}{2592 m_{1}{ }^{11 / 6}}
\end{aligned}
$$

\section{Equation of Fluid Flow}

The model and equations mentioned above are applied to a 
turbulent jet flow. The flow is assumed to be incompressible, and the continuity equation and Reynolds-averaged NavierStokes equations are:

$\nabla \cdot \overline{\boldsymbol{u}}=0$

$\frac{\partial \overline{\boldsymbol{u}}}{\partial t}+\overline{\boldsymbol{u}} \cdot \nabla \overline{\boldsymbol{u}}=-\frac{1}{\rho} \nabla \bar{p}+v \nabla^{2} \overline{\boldsymbol{u}}+\frac{1}{\rho} \nabla \cdot \mathbf{R}_{t}$

where $\bar{p}$ is the mean pressure; $\overline{\boldsymbol{u}}$ is the mean fluid velocity; $\rho$ is the fluid density, $v$ is the fluid kinematic viscosity and $\mathbf{R}_{t}$ is the Reynolds stress:

$\mathbf{R}_{t}=-\rho \overline{u_{i}^{\prime} u_{j}^{\prime}}=2 \rho v_{t}\left(\frac{\partial \bar{u}_{i}}{\partial x_{j}}+\frac{\partial \bar{u}_{j}}{\partial x_{i}}\right)-\frac{2}{3} \rho k \delta_{i j}$

where $u_{i}{ }^{\prime}$ and $u_{j}{ }^{\prime}$ represent the fluctuating fluid velocity; $\overline{u_{i}}$ and $\overline{u_{j}}$ represent the mean fluid velocity; $\delta_{i j}$ is Kronecker delta. Turbulent viscosity $v_{t}$ is given by $v_{t}=C_{\mu} k^{2} / \varepsilon$, where $k$ and $\varepsilon$ are the turbulent kinetic energy and turbulent dissipation rate, respectively. The corresponding equations are (Faghani et al., 2011; Ghahremanian and Moshfegh, 2014):

$\frac{\partial k}{\partial t}+\overline{\boldsymbol{u}} \cdot \nabla k-\nabla \cdot\left(v+\frac{v_{t}}{\sigma_{k}}\right) \nabla k=\frac{1}{\rho} \mathbf{R}_{t}: \nabla \overline{\boldsymbol{u}}-\varepsilon$

$\frac{\partial k}{\partial t}+\overline{\boldsymbol{u}} \cdot \nabla \varepsilon-\nabla \cdot\left(v+\frac{v_{t}}{\sigma_{e}}\right) \nabla \varepsilon=C_{\varepsilon 1} \frac{1}{\rho} \mathbf{R}_{t}: \nabla \overline{\boldsymbol{u}} \frac{\varepsilon}{k}-C_{\varepsilon 2} \frac{\varepsilon^{2}}{k}$

The values of the constants appearing in above equations are:

$C_{\mu}=0.09, C_{\varepsilon 1}=1.44, C_{\varepsilon 2}=1.92, \sigma_{k}=1, \sigma_{\varepsilon}=1.3$

\section{COMPUTATION SETTINGS}

Turbulent jet flow and coordinate system are shown in Fig. 1 where we extract a wedge domain from the cylinder based on the axisymmetric assumption. The tip of the wedge is the axis of the circular. A single layer of mesh is adopted to discretize the wedge domain, which converts a threedimensional axisymmetric problem into a two-dimensional problem. The included angle of two lateral sides is less than $5^{\circ}$.

The turbulent jet flow laden with aerosol particles is simulated numerically under different conditions as shown in Table 1. The initial values of $j^{\text {th }}$ moment and initial particle diameter are denoted by $m_{j 0}(j=0,1,2)$ and $d_{p 0}$, respectively. Computations are performed in OpenFOAM-5 using finite volume method. The pressure and mean velocity are coupled by the OpenFOAM PIMPLE algorithm. The Gauss limited linear difference scheme is adopted for the convective term in Eqs. (14), (16), (18) and (19).

\section{VALIDATIONS}

\section{Fluid Mean Velocity}

The turbulent jet flow of pure fluid is simulated first as a validation. The self-similarity of velocity profile in the fully developed zone far from the nozzle is a kind of properties of the turbulent jet flow (Pope, 2000). The self-preservation of the mean stream-wise velocity is shown in Fig. 2 where vertical coordinate is normalized by $U_{c l}$ (the mean velocity at center line) and horizontal coordinate is the radial location scaled by jet spreading half width $y / y_{1 / 2}$ (the radial coordinate where $U=1 / 2 U_{c l}$ in jet). We can see that there exists selfsimilarity of velocity profile at different stream-wise locations.

\section{Turbulent Kinetic Energy of Fluid}

Another validation we concerned is the turbulent kinetic energy as shown in Fig. 3 where the numerical results of pure fluid are plotted in solid-scatter line and experimental

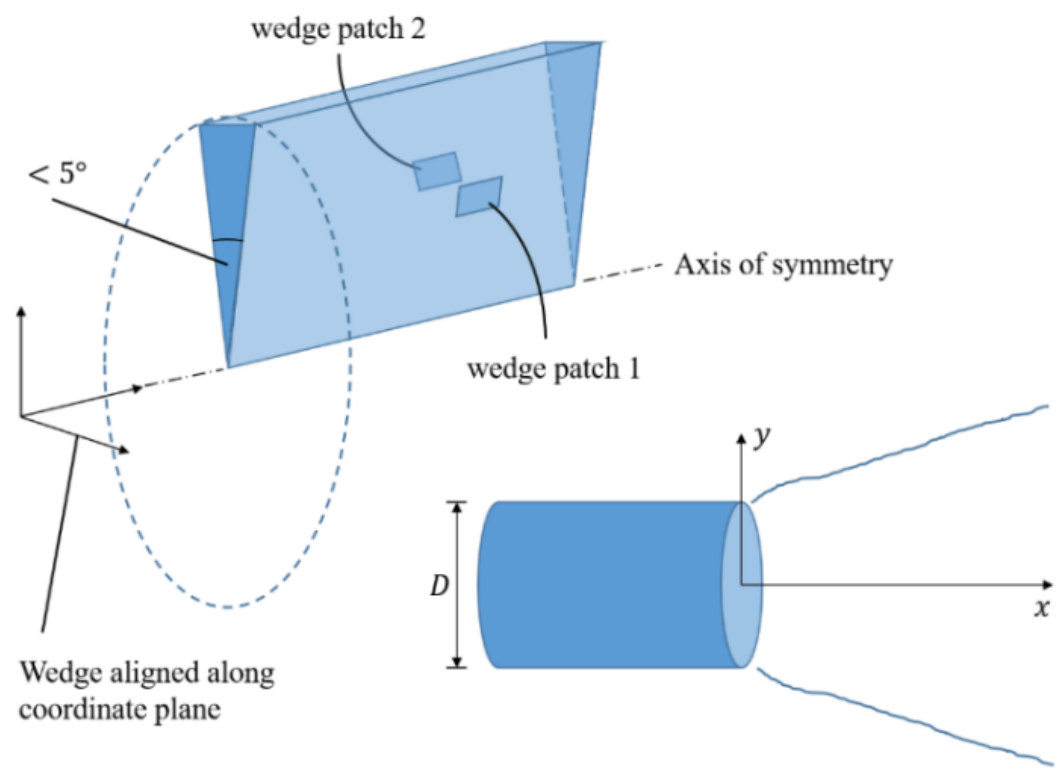

Fig. 1. The sketches of turbulent jet and the wedge-shaped computational domain. 
Table 1. Computational setting.

\begin{tabular}{lll}
\hline Mesh & Computational domain size & $X \times Y=100 D \times 25 D$ \\
Fluid phase & Grid size & Non-uniform: $0.01 D-0.3 D$ \\
& Inlet velocity $\left(U_{0}\right)$ & $10 \mathrm{~m} \mathrm{~s}^{-1}, 15 \mathrm{~m} \mathrm{~s}^{-1}, 30 \mathrm{~m} \mathrm{~s}^{-1}$ \\
& Inlet diameter $(D)$ & $0.01 \mathrm{~m}$ \\
Particle phase & $6667,10,000,20,000$ \\
& Reynolds number $(R e)$ & $3.6 \times 10^{-9} \mathrm{~m}$ \\
& Initial particle diameter $\left(d_{p 0}\right)$ & 1.00 \\
& $C_{M}=m_{00} m_{20} / m_{10}{ }^{2}$ & $1.80 \times 10^{-8}, 1.15 \times 10^{-8}, 8.15 \times 10^{-9}, 2.15 \times 10^{-9}, 9.75 \times 10^{-9}$ \\
& Inlet volume fraction $\left(m_{10}\right)$ & $\chi_{t}=0$, neglecting FCT \\
& Number concentration fluctuation & $\chi_{t}=k /\left(u_{i}^{2} / 2+k\right)$, present work \\
\hline
\end{tabular}

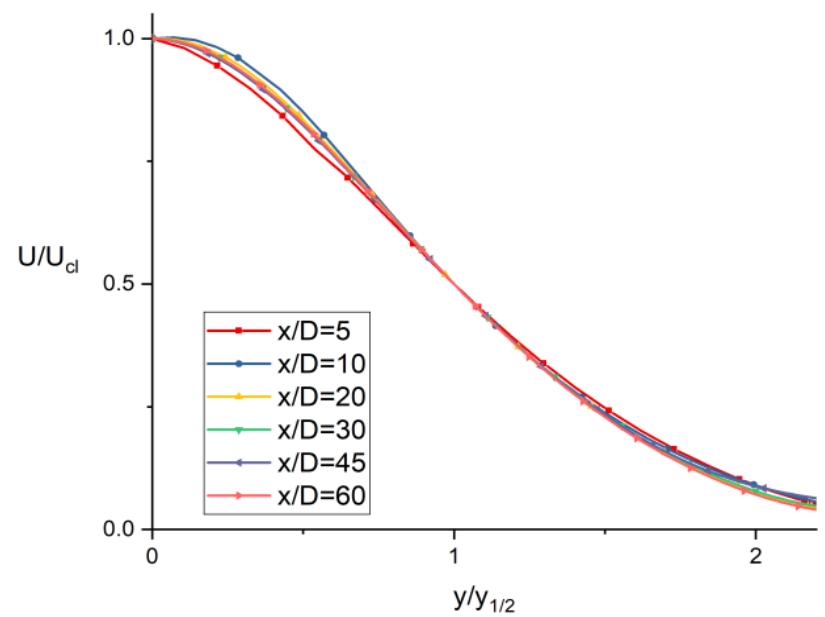

Fig. 2. The self-similarity curve of the mean stream-wise velocity.

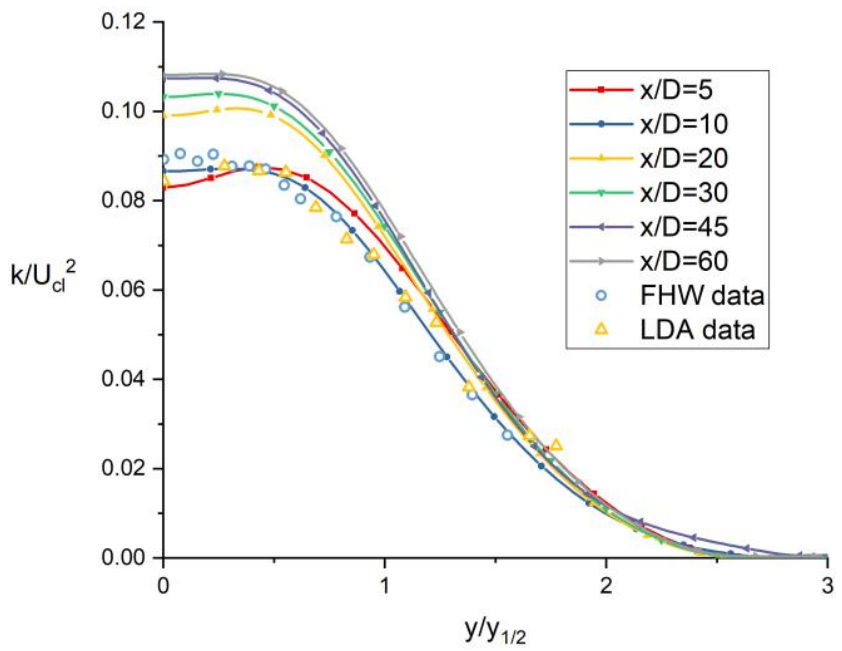

Fig. 3. Comparison of turbulent kinetic energy for the numerical and experimental results.

results (Hussein et al., 1994) measured with flying hot-wire (FHW) and burst-mode laser Doppler anemometer (LDA) also are given. It can be seen that the numerical results are in good agreement with the experimental data.

\section{Geometric Mean Diameter of Particle}

Geometric mean diameter as a function of total volume

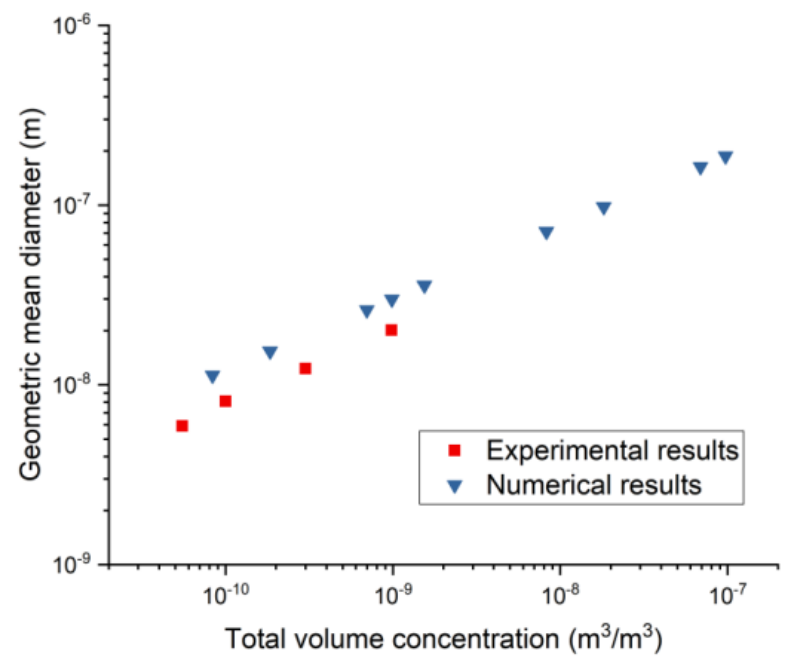

Fig. 4. Geometric mean diameter as a function of total volume concentration in $x D=60(R e=10,000)$.

concentration in $x D=60$ is shown in Fig. 4 where the experimental results (Chowdhury et al., 2017) are also given. We can see that two results are basically consistent.

\section{RESULTS AND DISCUSSIONS}

\section{Particle Number Concentration}

In order to compare the numerical results with the experimental ones, the total number concentration of particles as a function of total volume concentration in $x D=60$ is shown in Fig. 5 where both numerical and experimental results (Chowdhury et al., 2017) are given. It can be seen that the total number concentration decreases with increasing the total volume concentration, which is reasonable because of particle coagulation.

Fig. 6 shows the distribution of particle number concentration $m_{0} / m_{00}$ in the flow. We can see that the large values of $m_{0} / m_{00}$ occur in the area close to jet exit and center line, and the values decrease gradually along the flow and transverse directions.

Fig. 7 shows the variation of particle number concentration $m_{0} / m_{00}$ along the axial direction. Particle number concentration decreases rapidly along the axial direction because of flow convection, particle diffusion and coagulation, and the dilution of particulate jet flow with the ambient fluid. Comparing the cases with different initial mass concentration, it can be seen 


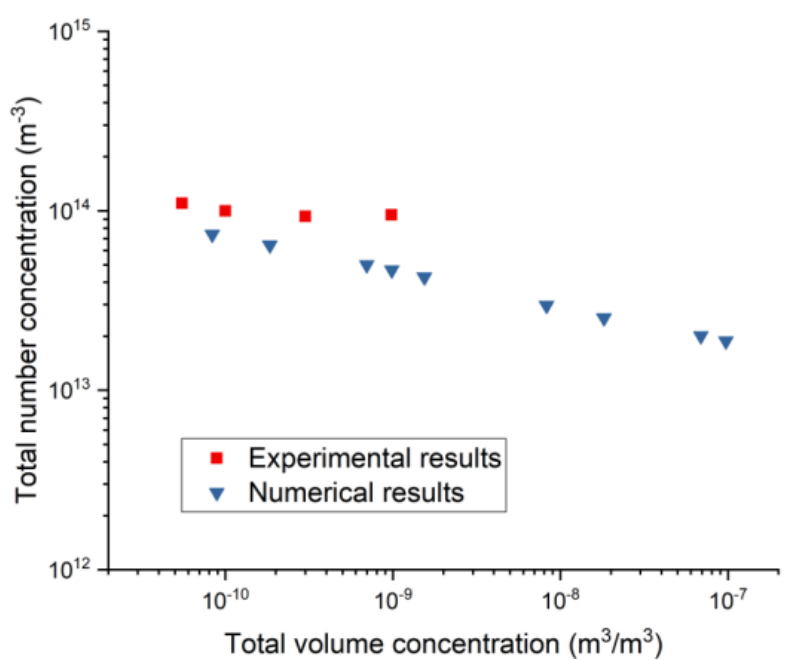

Fig. 5. Total number concentration as a function of total volume concentration in $x D=60(R e=10,000)$.

that higher initial mass concentration $m_{10}$ causes more frequent particle coagulation so that the number concentration is reduced faster along the axial direction. Comparing the cases with and without considering the FCT, we can see that the number concentration decreases with a same rate near the exit of the jet $(x / D<5)$ for both cases because the fluctuating concentration has an insignificant effect on the particle coagulation in this region. As the flow develops downstream, the difference in number concentration between the two cases is obvious, i.e., the number concentration with considering the FCT is less than that without considering the FCT because fluctuating concentration causes more frequent particle coagulation.

Variations of particle number concentration along the radial direction at different axial locations are plotted in Fig. 8 where the curves are totally different from those in Fig. 7. Particle number concentration decreases from the center of the jet to the outer edge first slowly and then sharply because of the rolling up of the primary vortex in the area near the edge. The region occupied with particles increases along the axial direction due to the influence of the vortices which act to increase the particle diffusion. Particle number concentration decreases in the jet core as the jet is developing. Within the region near the jet nozzle, particle number concentration decreases rapidly due to the coagulation is strong for the small particles. The phenomenon that particle number

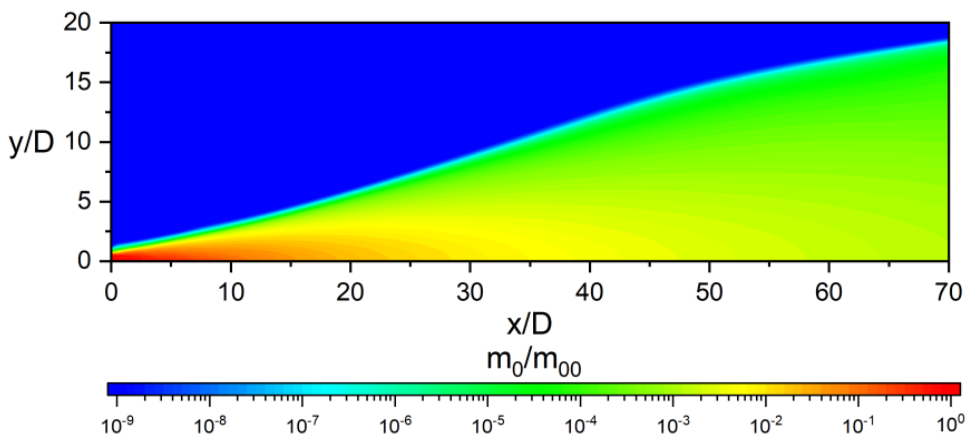

Fig. 6. The contour of $m_{0} / m_{00}\left(m_{0}=9.75 \times 10^{-10}, R e=10,000\right)$.

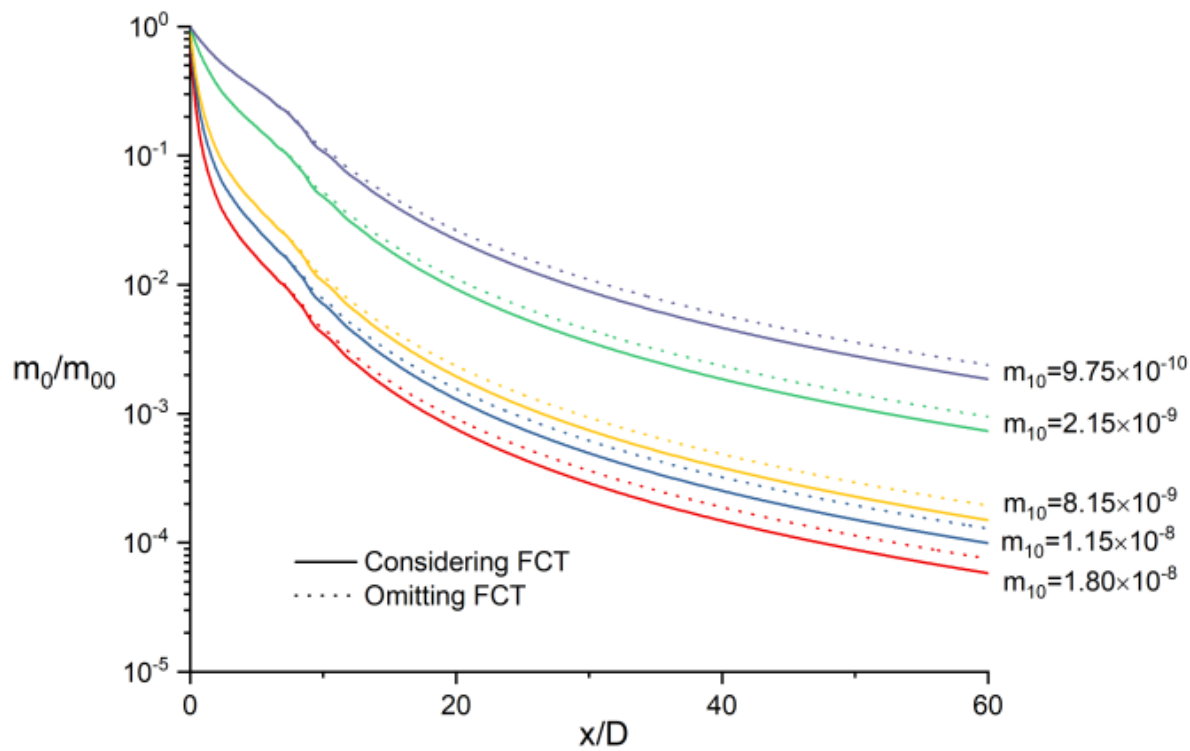

Fig. 7. Variation of particle number concentration $(R e=10,000)$. 


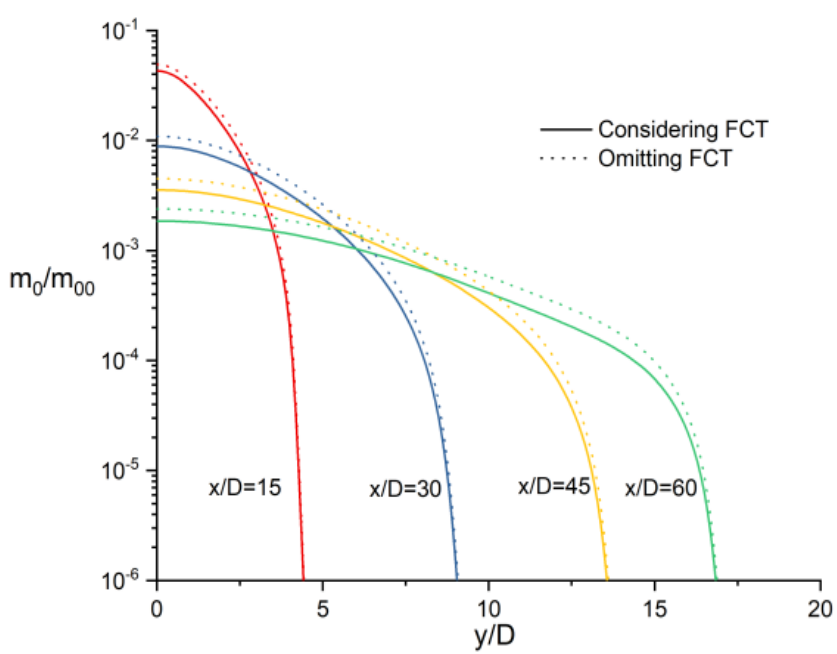

Fig. 8. Variation of particle number concentration along the radial direction $\left(m_{10}=9.75 \times 10^{-10}, R e=10,000\right)$.

concentration with considering the FCT is less than that without considering the FCT is more obvious in the area near the center line, which is consistent with the fact that the turbulent kinetic energy is larger in the area near the center line as shown in Fig. 3.

\section{Particle Mass Concentration}

Distribution of particle mass concentration $m_{1} / m_{10}$ in the flow is shown in Fig. 9, and variations of particle mass concentration along axial and lateral directions are shown in Fig. 10. It can be seen that the large values of particle mass concentration occur in the area close to jet exit and center line. The difference for two curves in Fig. 10 is obvious. As the flow is developing, aerosol particles diffuse across the fluid stream while diluting in the jet core, but the total particle mass remains unchanged in the whole flow field. As shown in Fig. 10(a), the particle mass concentration remains unchanged near the jet nozzle, and then decreases first rapidly and then slowly along the axial direction. However, particle mass concentration decreases from the center region to the jet edge with an almost constant rate as shown in Fig. 10(b).

\section{Particle Size}

Aerosol particle coagulation results in the growth of particle size. The geometric average diameter of particle $\left(d_{p}\right)_{g}$ can be determined by the geometric average volume:

$\left(d_{p}\right)_{g}=\left(v_{g}\right)^{1 / 3}$

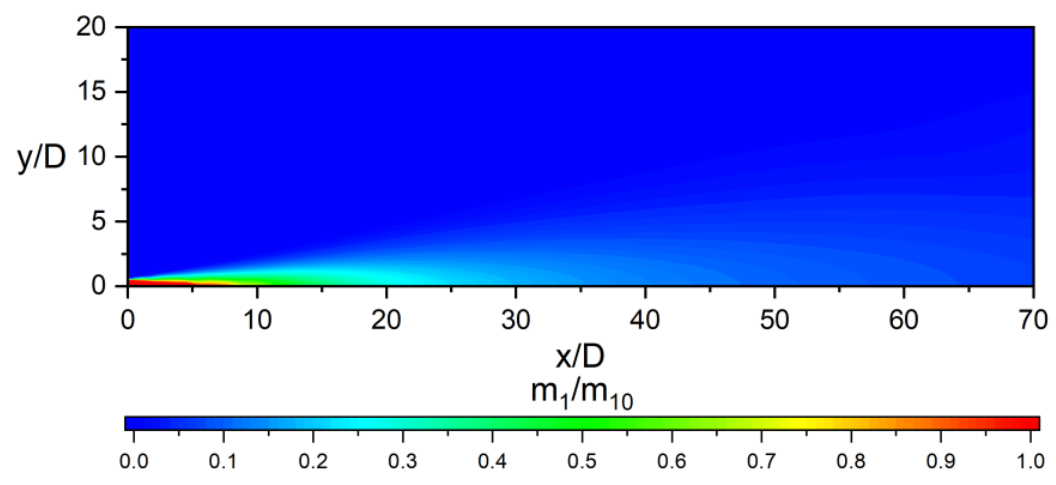

Fig. 9. The contour of $m_{1} / m_{10}\left(m_{10}=9.75 \times 10^{-10}, R e=10,000\right)$.

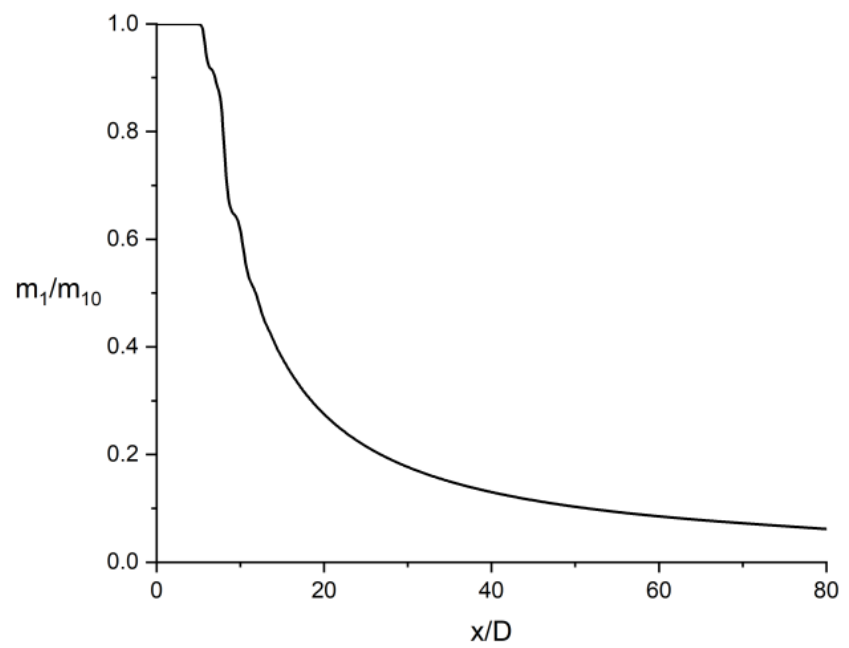

(a)

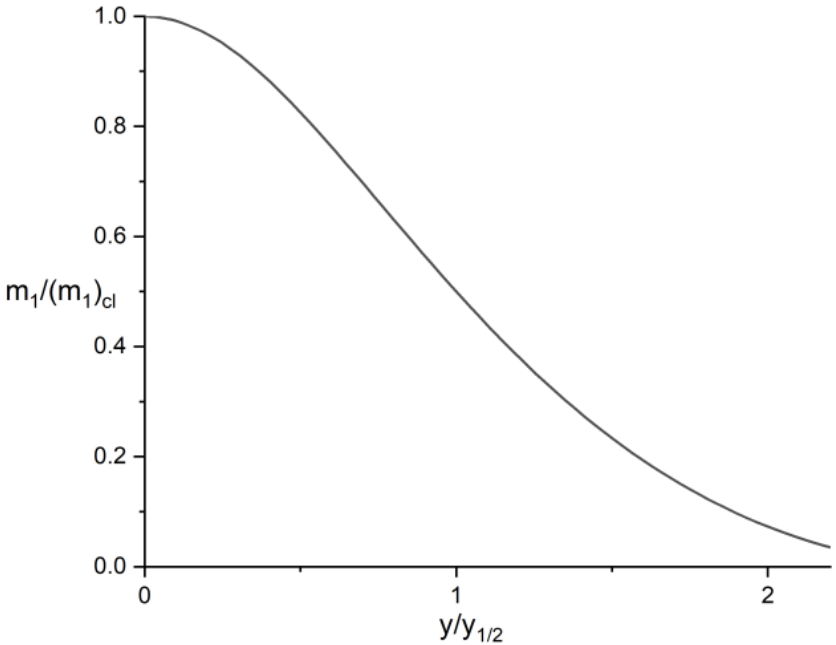

(b)

Fig. 10. Variation of particle mass concentration $m_{1}$ along (a) the axial direction and (b) the radial direction. 
while geometric average volume of particle is given by (Lee et al., 1984):

$v_{g}=\frac{\left(m_{1} / m_{10}\right)^{2}}{\left(m_{0} / m_{00}\right)^{3 / 2}\left(m_{2} / m_{20}\right)^{1 / 2}}$

The second moment $m_{2}$ appears in Eq. (21). Fig. 11 shows the distribution of $m_{2} / m_{20}$ in the flow.

Fig. 12 shows that geometric average diameter of particle increases along the axial direction due to particle coagulation. In the cases with higher initial mass concentration, particle diameter increases rapidly because of more frequent particle coagulation. Particle diameter with considering the FCT is larger than that without considering the FCT because fluctuating concentration causes more frequent particle coagulation.

Fig. 13 shows the variations of geometric average diameter of particle along the radial direction at different axial locations. Particle diameter increases slightly from the center of the jet to the outer edge and is reduced to 1 at the outer edge. The largest values of particle size are found on the interface of the jet and the ambient fluid. Particle diameter with considering the FCT is larger than that without considering the FCT, which is more obvious along the radial direction than that along the axial direction as shown in Fig. 12.

\section{Standard Variance of Particle Volume}

Another important parameter for particle size distribution is the geometric standard deviation $\sigma_{g}$ which is given by (Lee et al., 1984):

$\ln ^{2} \sigma_{g}=\frac{1}{9} \ln \left[\frac{\left(m_{0} / m_{00}\right)\left(m_{2} / m_{20}\right)}{\left(m_{1} / m_{10}\right)^{2}}\right]$

For the Brownian coagulation in the free molecule regime, the geometric standard deviation $\sigma_{g}$ reaches the asymptotic value of $\sigma_{\infty}=1.355$ when the particle system with the lognormal size distribution reaches the self-preserving size spectrum (Lee et al., 1984; Chen et al., 2014).

The geometric standard deviation of particles along the axial direction is shown in Fig. 14(a). The value of $\sigma_{g}$ grows rapidly near the exit of the jet $(x / D<10)$, and then tends to the asymptotic value of $\sigma_{\infty}=1.355$. The geometric standard deviation with higher initial mass concentration and with considering the FCT reaches the asymptotic value faster. A magnified diagram is plotted in Fig. 14(b) where the oscillation process of the curve can be seen in the region of $x / D<20$.

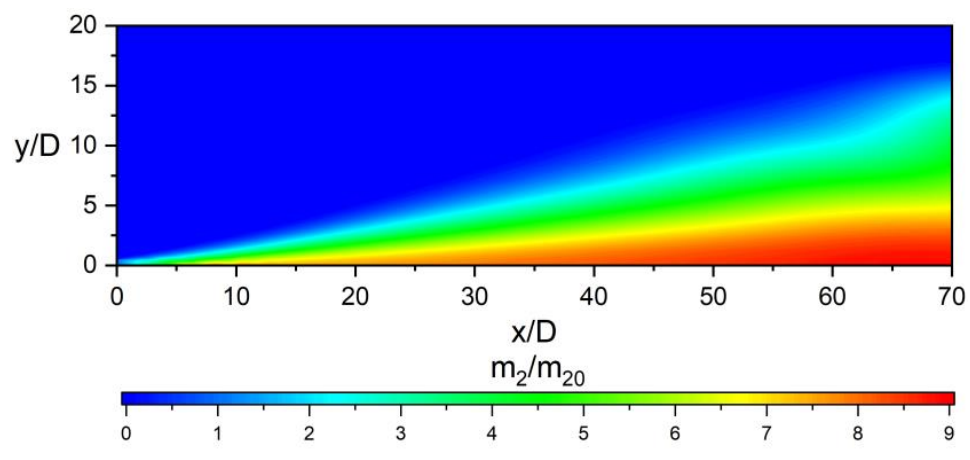

Fig. 11. The contour of $m_{2} / m_{20}\left(m_{10}=9.75 \times 10^{-10}, R e=10,000\right)$.

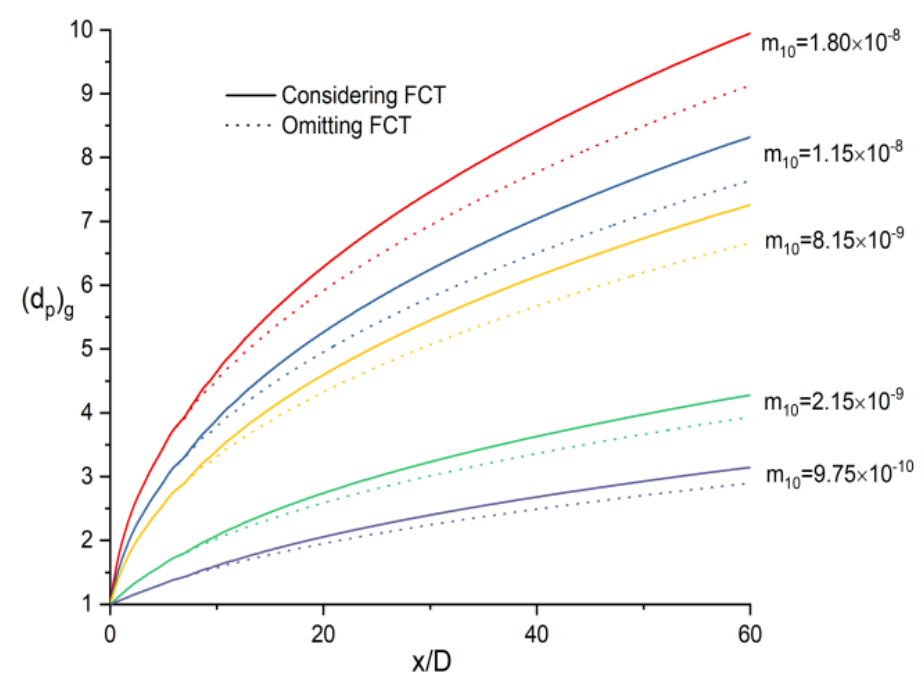

Fig. 12. Variation of geometric average diameter of particle along the axial direction $(\operatorname{Re}=10,000)$. 


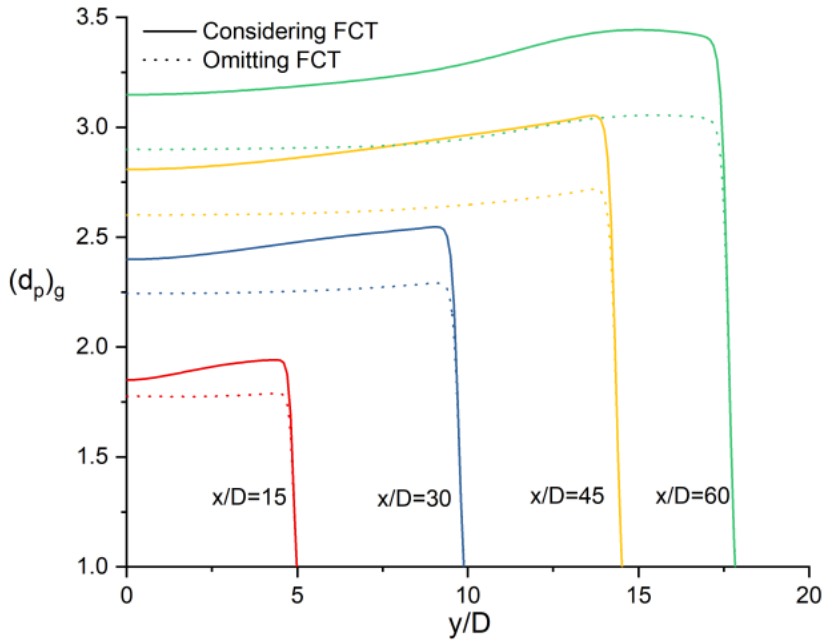

Fig. 13. Variation of geometric average diameter along the radial direction $\left(m_{10}=9.75 \times 10^{-10}, R e=10,000\right)$.

Fig. 15 shows the variations of geometric standard deviation of particles along the radial direction at different axial locations. The geometric standard deviation almost increases gradually from the center of the jet to the outer edge, and sharply near the outer edge. The highest values of geometric standard deviation are found near the interface of the jet region and the ambient fluid because the large velocity gradient near the interface makes the particles diffuse outwards, resulting in a rapid increase of $\sigma_{g}$. Besides, the geometric standard deviation with considering the FCT is larger than that without considering the FCT.

\section{CONCLUSIONS}

We apply the Reynolds averaging method to derive the averaged particle general dynamic equation (APGDE), which models the effect of fluctuations in aerosol concentrations on coagulation, referred to as the fluctuating coagulation term (FCT), based on the turbulent kinetic energy. The APGDE is numerically solved using the Taylor-series expansion method of moments for a turbulent jet; the equation is then validated by comparing some of the results with experimental values. Analyzing the distribution and evolution of the aerosol particle number concentration, mass concentration, mean diameter and geometric standard deviation both when the FCT is incorporated and when it is excluded, we arrive at the following conclusions.

The particle number concentration decreases rapidly along the axial direction, especially with high initial mass concentrations, whereas it decreases from the center to the outer edge, first slowly and then sharply, along the radial direction. The mass concentration remains unchanged near the jet nozzle but then decreases, first rapidly and then slowly, along the axial direction; however, along the radial direction, it decreases from the center to the outer edge at an almost constant rate. The particle diameter increases along the axial direction, and it increases from the center outward before decreasing to 1 at the outer edge along the radial direction. The largest values are found at the interface between the jet and the ambient fluid. The geometric standard deviation grows rapidly near the exit of the jet and then tends to the asymptotic value of 1.355 along the axial direction, increasing gradually at first from the center outward and then sharply near the outer edge.

The contribution of fluctuating concentrations to the coagulation becomes a factor when $x / D=5$ and reaches a stable state when $x / D=20$. As the flow develops downstream, the particle number concentration when incorporating the FCT is clearly lower than when excluding it; this difference becomes more prominent in the area near the center line. Additionally, incorporating the FCT produces a larger particle diameter, which is more obvious along the radial than the axial direction, and a greater geometric standard deviation, which reaches the asymptotic value more quickly. The

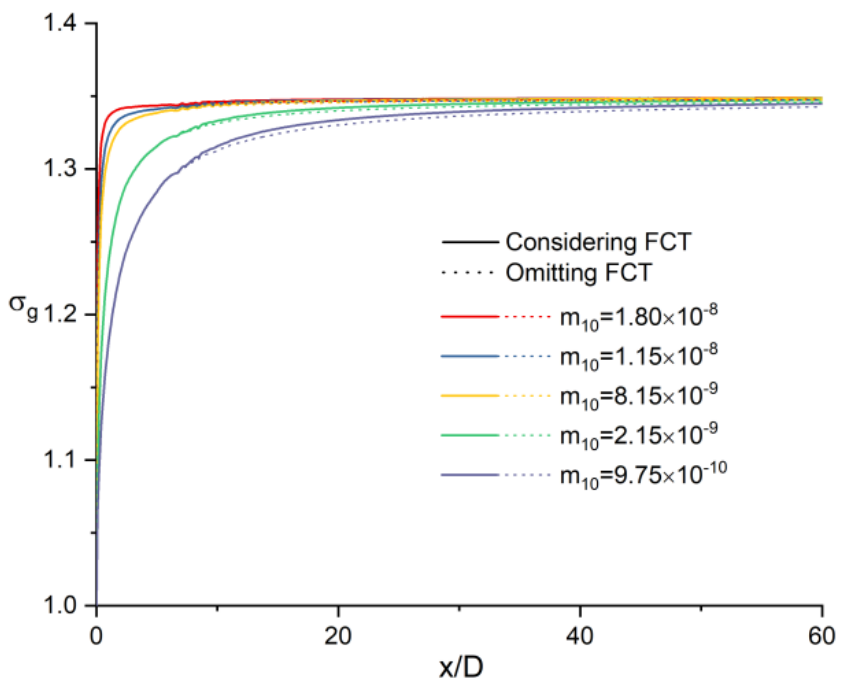

(a)

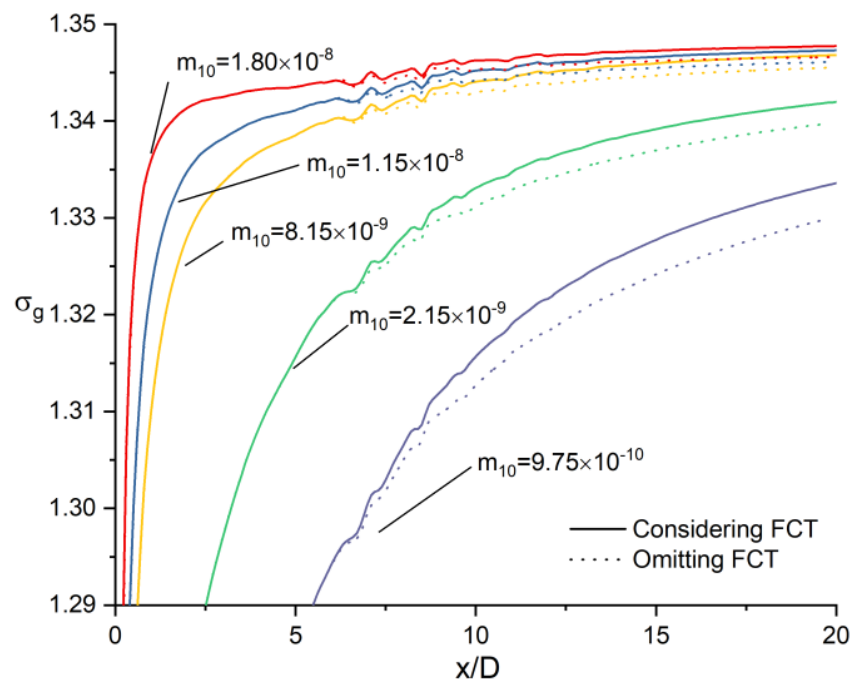

(b)

Fig. 14. (a) Variation of geometric standard deviation along the axial direction $(\operatorname{Re}=10,000)$ and (b) magnified diagram in $x / D<20$. 


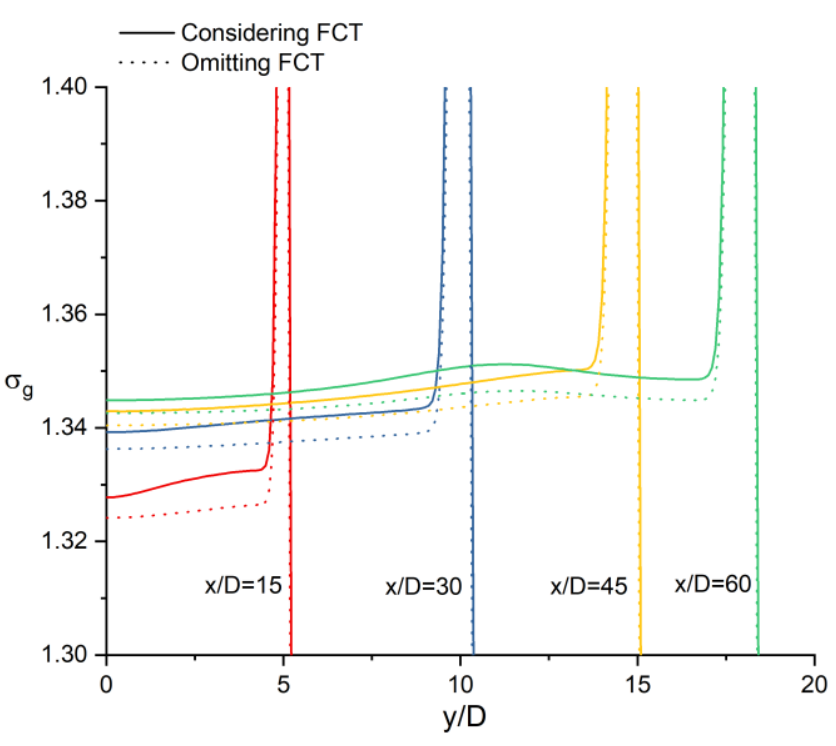

Fig. 15. Variation of geometric standard deviation along the radial direction $\left(m_{10}=9.75 \times 10^{-10}, R e=10,000\right)$.

fluctuating concentrations intensify the coagulation, reduce the number concentration and increase the mean diameter. Thus, such fluctuations in a turbulent jet cannot be neglected.

\section{ACKNOWLEDGMENTS}

This work was supported by the Major Program of the National Natural Science Foundation of China with Grant No. 91852102.

\section{REFERENCES}

Chan, T.L., Liu, S. and Yue, Y. (2018). Nanoparticle formation and growth in turbulent flows using the bimodal TEMOM. Powder Technol. 323: 507-517. https://doi.org/10.1016/j.powtec.2017.10.012

Chen, Z.L., Lin, J.Z. and Yu, M.Z. (2014). Asymptotic behavior of the Taylor-expansion method of moments for solving a coagulation equation for Brownian particles. Particuology 14: 124-129. https://doi.org/10.1016/j.partic. 2013.04.012

Chen, Z.L., Yuan, F.Y. and Jiang, R.J. (2015). Different approximate models of the sectional method for nanoparticle Brownian coagulation. Int. J. Numer. Methods Heat Fluid Flow 25: 438-448. https://doi.org/10.1108/hff-04-20130135

Chowdhury, S., Boyette, W.R. and Roberts, W.L. (2017). Time-averaged probability density functions of soot nanoparticles along the centerline of a piloted turbulent diffusion flame using a scanning mobility particle sizer. J. Aerosol Sci. 106: 56-67. https://doi.org/10.1016/j.jaero sci.2016.10.012

Chun, J., Koch, D.L., Rani, S.L., Ahluwalia, A. and Collins, L.R. (2005). Clustering of aerosol particles in isotropic turbulence. J. Fluid Mech. 536: 219-251. https://doi.org/ 10.1017/s0022112005004568

Das, S. and Garrick, S.C. (2010). The effects of turbulence on nanoparticle growth in turbulent reacting jets. Phys. Fluids 22: 103303. https://doi.org/10.1063/1.3486203

Faghani, E., Saemi, S.D., Maddahian, R. and Farhanieh, B. (2011). On the effect of inflow conditions in simulation of a turbulent round jet. Arch. Appl. Mech. 81: 14391453. https://doi.org/10.1007/s00419-010-0494-8

Friedlander, S.K. (2000). Smoke, dust, and haze: Fundamentals of aerosol dynamics. 2nd ed. Oxford University Press, New York.

Gan, F., Lin, J.Z. and Yu, M.Z. (2010). Particle size distribution in a planar jet flow undergoing shear-induced coagulation and breakage. J. Hydrodyn. 22: 445-455. https://doi.org/10.1016/s1001-6058(09)60076-7

Ghahremanian, S. and Moshfegh, B. (2014). Evaluation of RANS models in predicting low Reynolds, free, turbulent round jet. J. Fluids Eng. 136: 11201. https://doi.org/10.11 $15 / 1.4025363$

Hussein, H.J., Capp, S.P. and George, W.K. (1994). Velocity measurements in a high-Reynolds-number, momentum-conserving, axisymmetric, turbulent jet. $J$. Fluid Mech. 258: 31-75. https://doi.org/10.1017/s002211 209400323X

Lai, Y., Liu, Y.Y., Yu, M.Z., Wang, L.N., Liu, J. and Li, Q. (2018). A new mathematical scheme for approximating the overall aerosol extinction coefficient during brownian coagulation. Aerosol Air Qual. Res. 18: 2895-2905. https://doi.org/10.4209/aaqr.2018.01.0021

Lee, K.W., Chen, J. and Gieseke, J.A. (1984). Log-normally preserving size distribution for Brownian coagulation in the free-molecule regime. Aerosol Sci. Technol. 3: 53-62. https://doi.org/10.1080/02786828408958993

Lesniewski, T.K. and Friedlander, S.K. (1998). Particle nucleation and growth in a free turbulent jet. Proc. R. Soc. London A 454: 2477-2504. https://doi.org/10.1098/rspa. 1998.0267

Lin, J.Z., Pan, X., Yin, Z.Q. and Ku, X.K. (2016). Solution of general dynamic equation for nanoparticles in turbulent flow considering fluctuating coagulation. Appl. Math. Mech. 37: 1275-1288. https://doi.org/10.1007/s10483016-2131-9

Lin, J.Z., Yu, M.Z, and Nie, D.M. (2016). On the nanoparticulate flow. J. Hydrody. 28: 961-970. https://doi.org/10.1016/s1001-6058(16)60697-2

Liu, H.M. and Chan, T.L. (2018). Differentially weighted operator splitting Monte Carlo method for simulating complex aerosol dynamic processes. Particuology 36: 114-126. https://doi.org/10.1016/j.partic.2017.04.002

Liu, S. and Chan, T.L. (2017). A coupled CFD-Monte Carlo method for simulating complex aerosol dynamics in turbulent flows. Aerosol Sci. Technol. 51: 269-281. https://doi.org/10.1080/02786826.2016.1260087

Loeffler, J., Das, S. and Garrick, S.C. (2011). Large eddy simulation of titanium dioxide nanoparticle formation and growth in turbulent jets. Aerosol Sci. Technol. 45: 616628. https://doi.org/10.1080/02786826.2010.551147

Pope, S.B. (2000). Turbulent flows. Cambridge University Press, New York.

Pratsinis, S. (1988). Simultaneous nucleation, condensation, and coagulation in aerosol reactors. J. Colloid Interface 
Sci. 124: 416-427. https://doi.org/10.1016/0021-9797(88) 90180-4

Reade, W.C. and Collins, L.R. (2000). Effect of preferential concentration on turbulent collision rates. Phys. Fluids 12: 2530-2540. https://doi.org/10.1063/1.1288515

Rittler, A., Deng, L., Wlokas, I. and Kempf, A.M. (2017). Large eddy simulations of nanoparticle synthesis from flame spray pyrolysis. P. Combust. Inst. 36: 1077-1087. https://doi.org/10.1016/j.proci.2016.08.005

Yin, Z.Q. and Liu, H.J. (2014). Numerical simulation of nanoparticles diffusion and coagulation in a twin- jet via a TEMOM method. Int. J. Numer. Methods Heat Fluid Flow 24: 1312-1320. https://doi.org/10.1108/hff-04-20130143

Yu, M.Z., Lin, J.Z., Chen, L.H. and Chan, T.L. (2006). Large eddy simulation of a planar jet flow with nanoparticle coagulation. Acta Mech. Sin. 22: 293-300. https://doi.org/10.1007/s10409-006-0011-Z

Yu, M.Z., Lin, J.Z. and Chan, T.L. (2008). A new moment method for solving the coagulation equation for particles in Brownian motion. Aerosol Sci. Technol. 42: 705-713. https://doi.org/10.1080/02786820802232972

Zaichik, L.I. and Solov'Ev, A.L. (2002). Collision and coagulation nuclei under conditions of Brownian and turbulent motion of aerosol particles. High Temp. 40: 422-427. https://doi.org/10.1023/A:1016072226148

Zhou, K. and Chan, T.L. (2011). Simulation of homogeneous particle nucleation in a free turbulent jet. Aerosol Sci. Technol. 45: 973-987. https://doi.org/10.1080/02786826. 2011.572935

Zhu, R.R., Zhang, Y.Y., Yuan, Y. and Li, S.Q. (2018). Deposition loss of particles in the sampling lines of continuous emission monitoring system (CEMS) in coalfired power plants. Aerosol Air Qual. Res. 18: 1483-1492. https://doi.org/10.4209/aaqr.2017.11.0523

Received for review, November 4, 2019

Revised, January 9, 2020

Accepted, February 24, 2020 